\title{
El plutón anorogénico Chinquilchoro del Pérmico medio: un caso de zonación concéntrica normal en su parte meridional, norte de Chile
}

\author{
*Hans Niemeyer ${ }^{1}$, Carolina Castillo ${ }^{1}$ \\ I Departamento de Ciencias Geológicas, Universidad Católica del Norte, Angamos 0610, Antofagasta, Chile. \\ hansn@ucn.cl; castillo.orrego@yahoo.com \\ *Autor para correspondencia: hansn@ucn.cl; hansniemeyer.r@gmail.com
}

\begin{abstract}
RESUMEN. La parte meridional del plutón Chinquilchoro, del Pérmico medio, presenta una zonación textural y mineralógica y se puede dividir en dos litofacies aproximadamente concéntricas: una litofacies A, melanocrática, externa, y una litofacies $\mathrm{B}$, leucocrática, interna. La primera consiste en diorita cuarcífera y monzodiorita cuarcífera, en tanto que la segunda tiene composición en el límite entre monzodiorita cuarcífera y monzonita cuarcífera. El contacto entre ambas litofacies es difuso y transicional, y las dos son calcoalcalinas, metaluminosas y férricas. La coexistencia de ambas litofacies se puede explicar por cristalización fraccionada a partir de un magma parental único en un ambiente tectónico anorogénico.
\end{abstract}

Palabras clave: Plutón pérmico zonado, Metaluminoso, Anorogénico.

\begin{abstract}
The Middle Permian Chinquilchoro anorogenic pluton: a case of normal concentric zonation in its southern part, northern Chile. The southern part of the Mid-Permian Chinquilchoro pluton consits of two approximately concentric lithofacies: an A lithofacies, external melanocratic and a leucocratic internal B lithofacies. The A lithofacies is formed by quartz diorite and quartz monzonite, and the B lithofacies lies in the limit between quartz monzodiorite and quartz monzonite. The contact between the two lithofacies is transitional and difuse. The two lithofacies are calcalkaline, metaluminous and ferric. The coexistence of both lithofacies can be explained by fractional crystallization from the same parental magma in an anorogernic tectonic environment.
\end{abstract}




\section{Introducción}

Algunos plutones se caracterizan por presentar una zonación concéntrica normal según sea su litología externa más máfica que la interna (Nédélec y Bouchez, 2015). Esta nota se refiere a la parte meridional del plutón Chinquilchoro. Este tiene una edad pérmica media y se ubica en el cordón del mismo nombre, al sur del salar de Atacama (Fig. 1), norte de Chile. Presenta una zonación concéntrica normal y tiene una sección semicircular con una superficie expuesta de $c a$. $28 \mathrm{~km}^{2}$. Allí afloran dos litofacies: una melanocrática, externa (A), y otra leucocrática, interna (B) (Fig. 2). El propósito de este trabajo es estudiar la génesis de la zonación y el ambiente tectónico de emplazamiento del plutón Chinquilchoro.

\section{Metodología}

El trabajo de campo incluye la cartografía del plutón, identificando las diferentes litofacies y el muestreo de estas. Durante el levantamiento cartográfico se recolectaron 19 muestras de ambas litofacies, las que fueron estudiadas por medio de microscopía estándar y analizadas con el objetivo de determinar su composición química. Para la determinación de la plagioclasa, el clinopiroxeno y la hornblenda, así como de las texturas, se utilizó un microscopio petrográfico disponible en el Departamento de Ciencias Geológicas de la Universidad Católica del Norte. También se efectuó su estudio por medio de microsonda electrónica en el Instituto de Geología Económica Aplicada de la Universidad de Concepción. Los análisis químicos de elementos mayores de las 19 muestras (Tabla 1) fueron realizados por el método de fluorescencia de rayos $\mathrm{X}$ mediante briquetas de polvo de roca prensado en el Laboratorio de Geoquímica de la Universidad Católica del Norte. Las muestras fueron colectadas en orientación noreste, según tres perfiles que cruzan transversalmente la parte meridional del plutón (Fig. 2 ).

\section{Edad y relaciones de contacto}

Se dispone de dos determinaciones de edad U-Pb por el método LA-ICP-MS en circones individuales, que se consideran edades de cristalización y son concordantes dentro de su error analítico. Una dió $266,1 \pm 3,0 \mathrm{Ma}$ en su sector norte, fuera del área cubierta por esta nota, y otra $267,3 \pm 2,5 \mathrm{Ma}$ en su sector meridional, dentro del área de estudio (Niemeyer, 2013). Dichas determinaciones son del Guadalupiano (Pérmico medio), lo que está de acuerdo con su relación de contacto intrusivo en lavas dacíticas de los Estratos de Cerro Negro, datadas en $272,7 \pm 1,6 \mathrm{Ma}$ por el método ${ }^{40} \mathrm{Ar} /{ }^{39} \mathrm{Ar}$ en biotita,

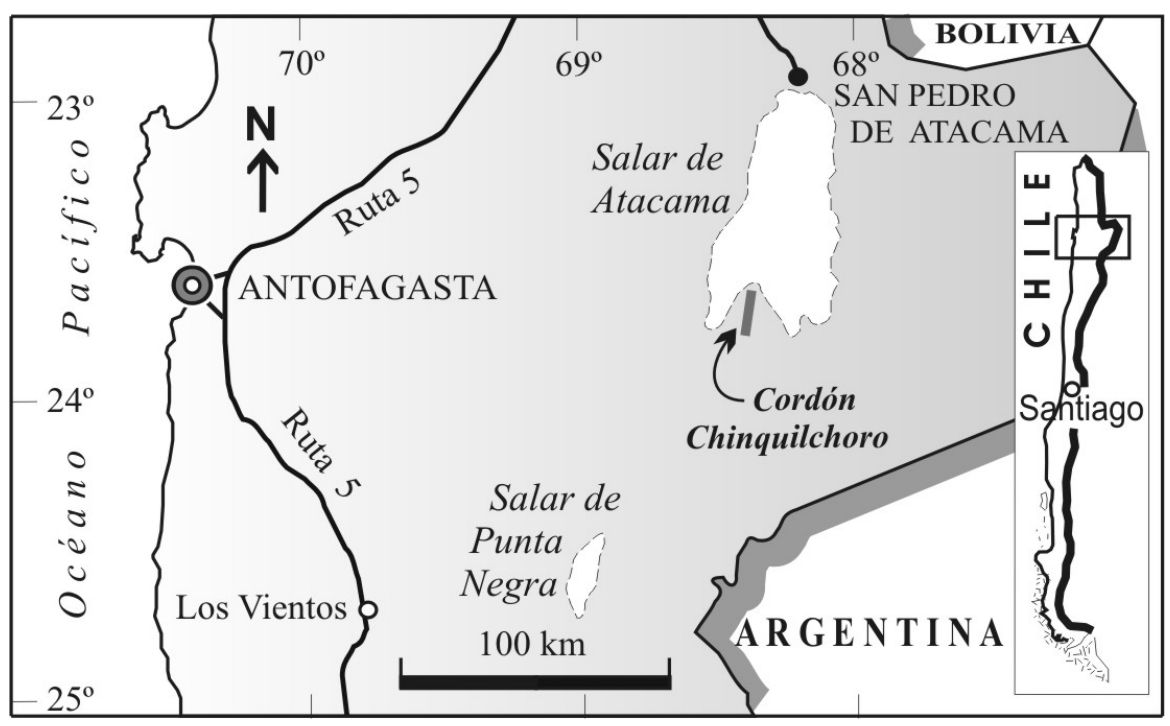

FIG. 1. Ubicación del Cordón Chinquilchoro, norte de Chile. 


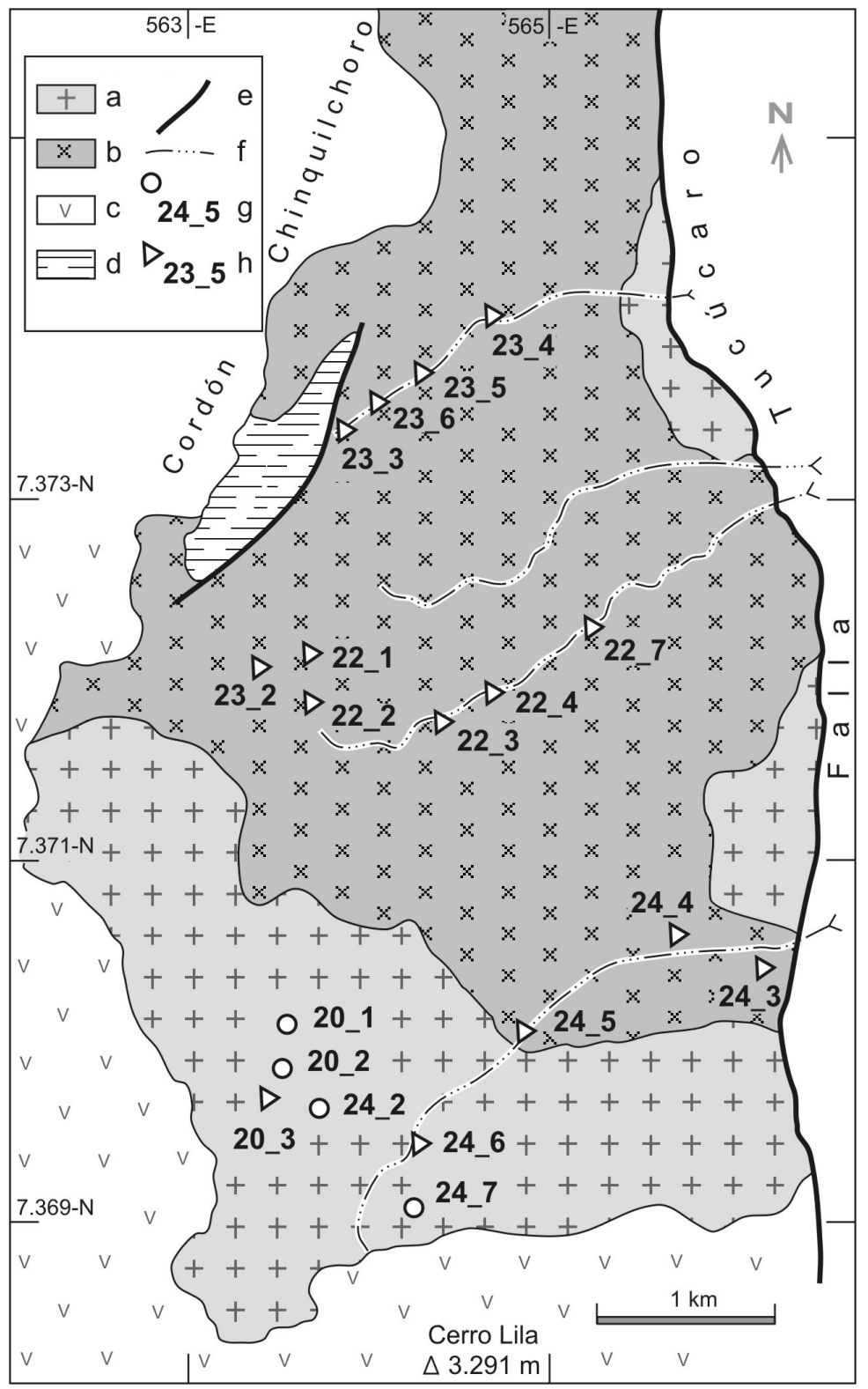

FIG. 2. Mapa geológico del extremo meridional del plutón Chinquilchoro, en el cual se han mapeado las dos litofacies que lo conforman. Se indica la ubicación de las diferentes muestras utilizadas en el presente estudio. a. Litofacies A. b. Litofacies B. c. Turbiditas y basaltos (Ordovícico). d. Cuarzoarenitas (Silúrico). e. Falla. f. Curso de quebradas. g. Muestras de litofacies A. h. Muestras de litofacies B.

fuera del área de estudio (Niemeyer, 2013). En su flanco suroccidental, el plutón Chinquilchoro está en contacto con rocas del Complejo ígneo-sedimentario del Cordón de Lila (Ordovícico; Niemeyer, 1989), en tanto que en su flanco oriental lo hace con la Falla Tucúcaro (Fig. 2).

\section{Clasificación petrográfica}

El plutón Chinquilchoro está formado por rocas de textura fanerítica hipidiomórfica de grano fino a medio (1-4 mm). Las dos litofacies que conforman la parte meridional del plutón, ocupan composiciones 
TABLA 1. CONTENIDO DE ELEMENTOS MAYORES (\% EN PESO): PLUTÓN CHINQUILCHORO.

\begin{tabular}{|c|c|c|c|c|c|c|c|c|c|c|c|}
\hline Muestra & $\begin{array}{l}\mathrm{SiO}_{2} \\
(\%)\end{array}$ & $\begin{array}{l}\mathrm{TiO}_{2} \\
(\%)\end{array}$ & $\begin{array}{c}\mathrm{Al}_{2} \mathrm{O}_{3} \\
(\%)\end{array}$ & $\begin{array}{c}\mathrm{Fe}_{2} \mathrm{O}_{3} \\
(\%)\end{array}$ & $\begin{array}{c}\mathrm{MnO} \\
(\%)\end{array}$ & $\begin{array}{c}\text { MgO } \\
(\%)\end{array}$ & $\begin{array}{c}\mathrm{CaO} \\
(\%)\end{array}$ & $\begin{array}{c}\mathrm{Na}_{2} \mathrm{O} \\
(\%)\end{array}$ & $\begin{array}{l}\mathrm{K}_{2} \mathrm{O} \\
(\%)\end{array}$ & $\begin{array}{c}\mathrm{P}_{2} \mathrm{O}_{5} \\
(\%)\end{array}$ & Total \\
\hline 20_1 & 53,68 & 0,74 & 17,26 & 9,26 & 0,16 & 2,55 & 10,39 & 4,05 & 1,41 & 0,49 & 99,99 \\
\hline 20_2 & 51,89 & 0,67 & 17,86 & 9,55 & 0,14 & 3,01 & 11,04 & 4,32 & 1,02 & 0,49 & 99,99 \\
\hline $20 \_3$ & 64,2 & 0,46 & 15,57 & 5,12 & 0,05 & 1,23 & 5,51 & 4,48 & 2,99 & 0,38 & 99,99 \\
\hline $22 \_1$ & 62,51 & 0,76 & 14,17 & 6,73 & 0,08 & 1,53 & 6,17 & 3,76 & 3,8 & 0,49 & 100 \\
\hline $22 \_2$ & 62,45 & 0,7 & 14,66 & 6,7 & 0,08 & 1,33 & 6,04 & 4,03 & 3,6 & 0,41 & 100 \\
\hline $22 \_3$ & 61,89 & 0,79 & 14,87 & 6,68 & 0,08 & 1,56 & 6,22 & 4 & 3,65 & 0,26 & 100 \\
\hline $22 \_4$ & 60,72 & 0,81 & 14,75 & 7,34 & 0,09 & 1,78 & 6,44 & 4,16 & 3,44 & 0,46 & 99,99 \\
\hline $22 \_7$ & 61,01 & 0,85 & 14,75 & 7,37 & 0,09 & 1,65 & 6,3 & 4,08 & 3,42 & 0,47 & 99,99 \\
\hline $23 \_2$ & 63,05 & 0,78 & 14,46 & 6,86 & 0,09 & 1,25 & 4,91 & 4,47 & 3,6 & 0,53 & 100 \\
\hline $23 \_3$ & 61,72 & 0,78 & 14,67 & 6,82 & 0,09 & 1,82 & 6,08 & 4,01 & 3,55 & 0,46 & 100 \\
\hline $23 \_4$ & 61,9 & 0,75 & 15,05 & 7,11 & 0,1 & 1,59 & 5,25 & 4,19 & 3,68 & 0,39 & 100,01 \\
\hline $23 \_5$ & 62,18 & 0,74 & 14,44 & 6,56 & 0,09 & 1,63 & 6,17 & 4 & 3,72 & 0,46 & 99,99 \\
\hline $23 \_6$ & 61,9 & 0,77 & 14,69 & 6,85 & 0,09 & 1,7 & 6,03 & 3,88 & 3,63 & 0,47 & 100,01 \\
\hline $24 \_2$ & 51,63 & 0,75 & 17,44 & 9,52 & 0,13 & 3,47 & 11,43 & 3,87 & 1,12 & 0,63 & 99,99 \\
\hline $24 \_3$ & 61,61 & 0,38 & 14,53 & 7,69 & 0,11 & 2,17 & 7,59 & 3,73 & 2,06 & 0,12 & 99,99 \\
\hline $24 \_4$ & 62,88 & 0,72 & 14,54 & 6,52 & 0,07 & 1,47 & 5,79 & 3,97 & 3,62 & 0,41 & 99,99 \\
\hline $24 \_5$ & 56,46 & 1,05 & 15,57 & 9,4 & 0,11 & 2,39 & 7,2 & 4,74 & 2,4 & 0,66 & 99,98 \\
\hline $24 \_6$ & 63,53 & 0,46 & 15,56 & 6,48 & 0,06 & 1,33 & 5,06 & 4,34 & 2,87 & 0,31 & 100 \\
\hline $24 \_7$ & 53,14 & 0,54 & 20,35 & 5,88 & 0,06 & 1,6 & 12,13 & 4,76 & 1,11 & 0,41 & 99,98 \\
\hline
\end{tabular}

distintas dentro del diagrama QAP de la clasificación de rocas plutónicas de Streckeisen (1974) (Fig. 3).

\subsection{Litofacies A}

Es una litofacies de color verde oscuro y se distribuye en la periferia, en el extremo sur del plutón. En el diagrama QAP ocupa el campo de la diorita cuarcífera, además de una muestra ubicada en el campo de la monzodiorita cuarcífera (Fig. 3). En esta litofacies, el feldespato dominante es la plagioclasa $(>70 \%)$, la cual tiene una zonación normal: bytownita, labradorita y andesina. El feldespato potásico es escaso (2\%) e intersticial, de tipo ortoclasa con textura pertítica.

Los minerales máficos corresponden a clinopiroxeno de tipo salita (12\%), hornblenda de tipo magnesio-hornblenda férrica $(5 \%)$ y biotita de color pardo (7\%). Los cristales de piroxeno están reemplazados en su parte externa por hornblenda, que es uralítica y no fibrosa, lo cual sugiere un proceso de cristalización normal (Heinrich, 1972).
A su vez, en la periferia de los cristales de hornblenda, se dispone una envoltura de biotita parda.

La magnetita está espacialmente relacionada con la hornblenda y con la biotita por medio de cristales anhedrales a subhedrales de hasta 1,2 $\mathrm{mm}$ de diámetro, a los cuales se asocian pequeños y abundantes cristales de apatito. Además de este último, otros minerales accesorios incluyen circón, esfeno $y$, muy escasamente, allanita.

En algunos lugares de esta litofacies se reconoció un bandeamiento de tipo schlieren (Fig. 4), cuyo manteo es de $c a .30^{\circ}$ hacia el centro del plutón.

\subsection{Litofacies B}

Esta litofacies es de color gris claro, y tiene una composición mineralógica que la ubica en el diagrama QAP (Fig. 3) entre monzodiorita cuarcífera y monzonita cuarcífera. Destaca la abundante cantidad de feldespato de tipo ortoclasa pertítica intersticial. Presenta intercrecimiento granofírico con el cuarzo, con un contenido que llega a 17\%. 


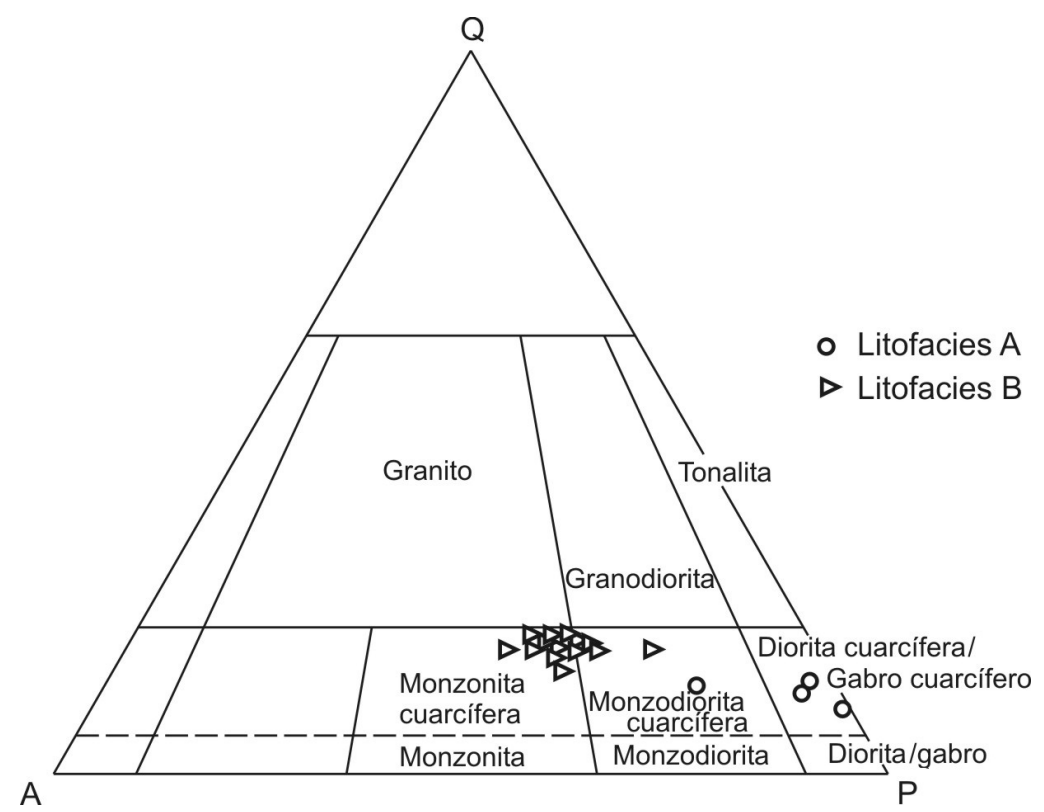

FIG. 3. Diagrama QAP (Q: cuarzo, A: feldespato alcalino, P: plagioclasa): clasificación mineralógica modal de las diferentes muestras de la parte meridional del plutón Chinquilchoro.

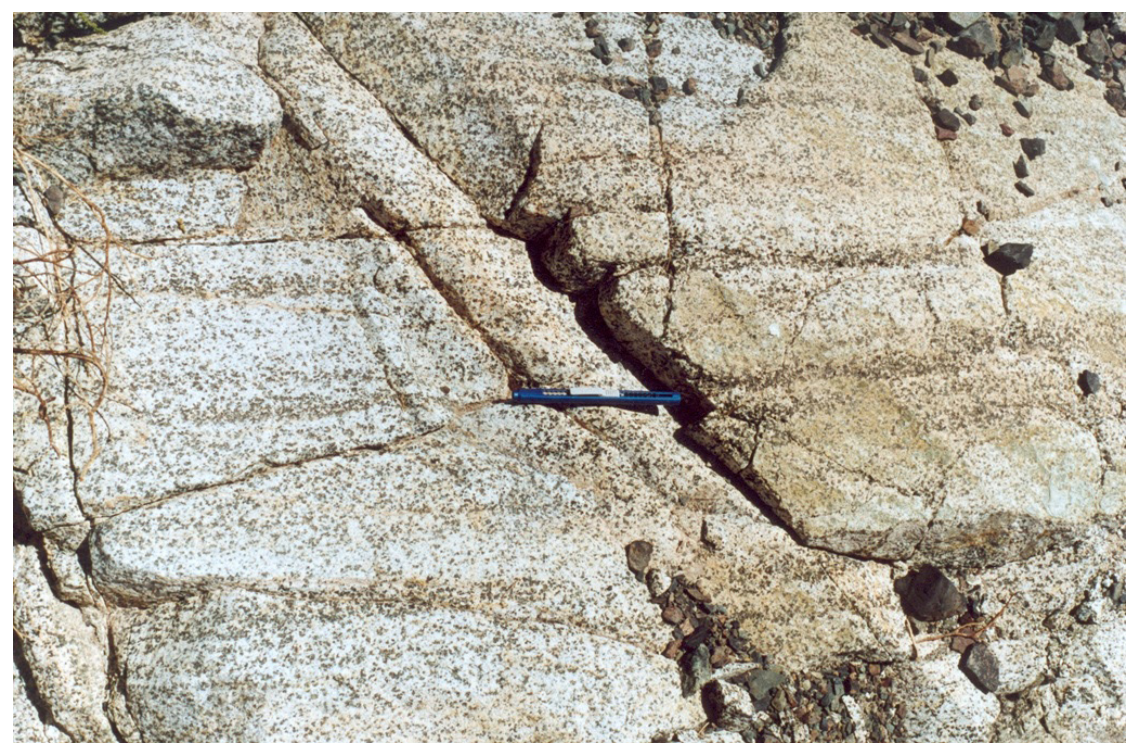

FIG. 4. Schlieren en un lugar externo de la parte meridional del plutón Chinquilchoro.

La cantidad de plagioclasa es notablemente menor (45\%) y es más sódica $\left(\mathrm{An}_{38-42}\right.$, andesina intermedia) que en la litofacies A. El piroxeno salítico es escaso o ausente, y cuando está presente es absorbido en su mayor parte por la hornblenda. La biotita alcanza casi el doble en cantidad (13\%) que en la litofacies A. El contacto entre ambas litofacies es difuso y transicional. 


\section{Clasificación química}

En el diagrama $\mathrm{Na}_{2} \mathrm{O}+\mathrm{K}_{2} \mathrm{O}-\mathrm{CaO}$ versus $\mathrm{SiO}_{2}$ (Frost et al., 2001), (Fig. 5), las rocas de la litofacies A se distribuyen a lo largo de la serie cálcica y las de la litofacies B lo hacen en el campo de la serie calcoalcalina, lo que explica su mineralogía diferente.

El índice molecular $\mathrm{ASI}=\mathrm{Al} /(\mathrm{Ca}-1,67 \mathrm{P}+\mathrm{Na}+\mathrm{K})$ es $<0$ con $(\mathrm{Na}+\mathrm{K})$ molecular $<\mathrm{Al}$ molecular, lo cual indica que todas las rocas son marcadamente metaluminosas (Frost et al., 2001).

En el diagrama $\mathrm{FeO} * /(\mathrm{FeO} *+\mathrm{MgO})$ versus $\mathrm{SiO}_{2}$ de Frost et al. (2001), la mayor parte de las rocas de ambas litofacies son férricas con excepción de la muestra 20_2 (Fig. 6).

\section{Discusión y conclusiones}

La distribución zonada de ambas litofacies en el plutón, con un borde diorítico y una zona central félsica sugiere que ellas se habrían formado por cristalización fraccionada in situ a partir del mismo magma parental (Barbey, 2009). La inclinación de los schlieren hacia el centro del plutón, así como su sección semicircular, sugieren una forma de embudo para esta parte del plutón Chinquilchoro (Marsh, 2015; Améglio y Vigneresse, 1999). La litofacies B, menos densa, se habría generado en la parte central de la cámara magmática y la $\mathrm{A}$, más densa, se habría segregado en el borde de esta (Marsh, 2015).
La cristalización inicial originó plagioclasa cálcica y augita, lo que caracteriza la litofacies diorítica y sugiere su origen temprano en la evolución del sistema magmático. En una etapa posterior, ocurrió la cristalización de anfíbola que reemplaza parcialmente al piroxeno y, seguidamente, la cristalización de biotita en torno a la anfíbola. La cristalización de los dos últimos minerales se entiende como una parte integral y final de una diferenciación netamente ígnea en presencia de agua (Shelley, 1993). En seguida se generó una abundante cristalización de magnetita asociada con apatito. En el sistema magnetita-apatito-diorita, la magnetita y el apatito se separan según dos fases inmiscibles a partir de un magma diorítico en un punto eutéctico, al final de su cristalización (Philpotts, 1967). Finalmente, ocurrió la cristalización residual de cuarzo junto con feldespato potásico, evidenciada por la textura granofírica intersticial de la litofacies B.

Este proceso de cristalización también se ve reflejado en los cambios composicionales observados entre ambas litofacies, con la litofacies félsica caracterizada por menores contenidos de $\mathrm{Fe}_{2} \mathrm{O}_{3}, \mathrm{CaO}$ y $\mathrm{MgO}$. Lo anterior es compatible con la cristalización inicial de piroxeno y plagioclasa cálcica, propia de la litofacies diorítica. Estos fundidos calcoalcalinos metaluminosos pudieron formarse por fusión parcial de corteza tonalítica o granodiorítica en condiciones de baja presión litostática (Frost y Frost, 2011).

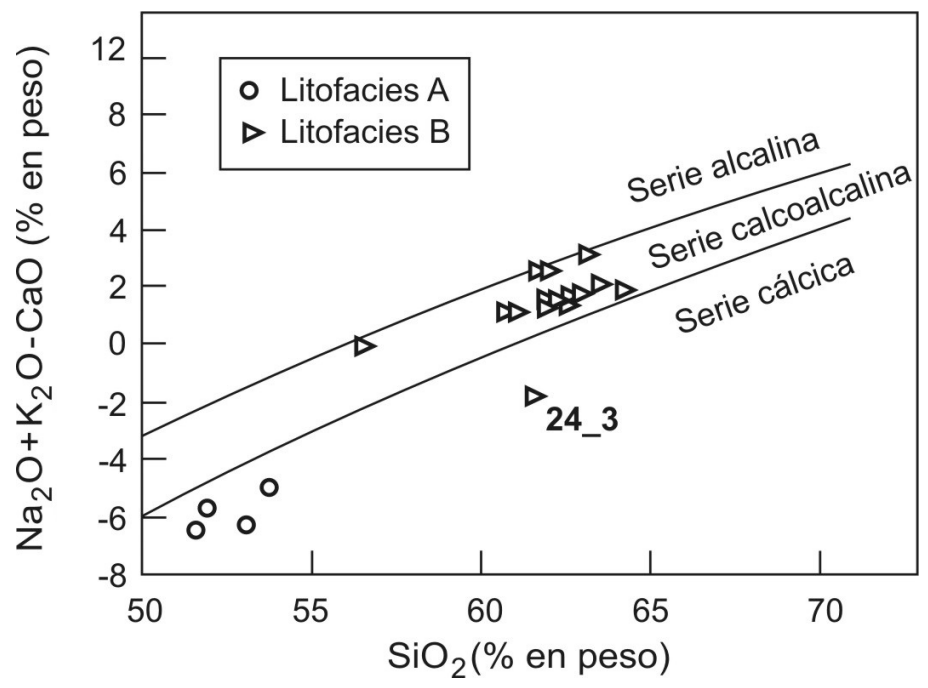

FIG. 5. Clasificación química de las muestras de la parte meridional del plutón Chinquilchoro en el diagrama $\mathrm{Na}_{2} \mathrm{O}+\mathrm{K}_{2} \mathrm{O}-\mathrm{CaO}$ versus $\mathrm{SiO}{ }_{2}$. 


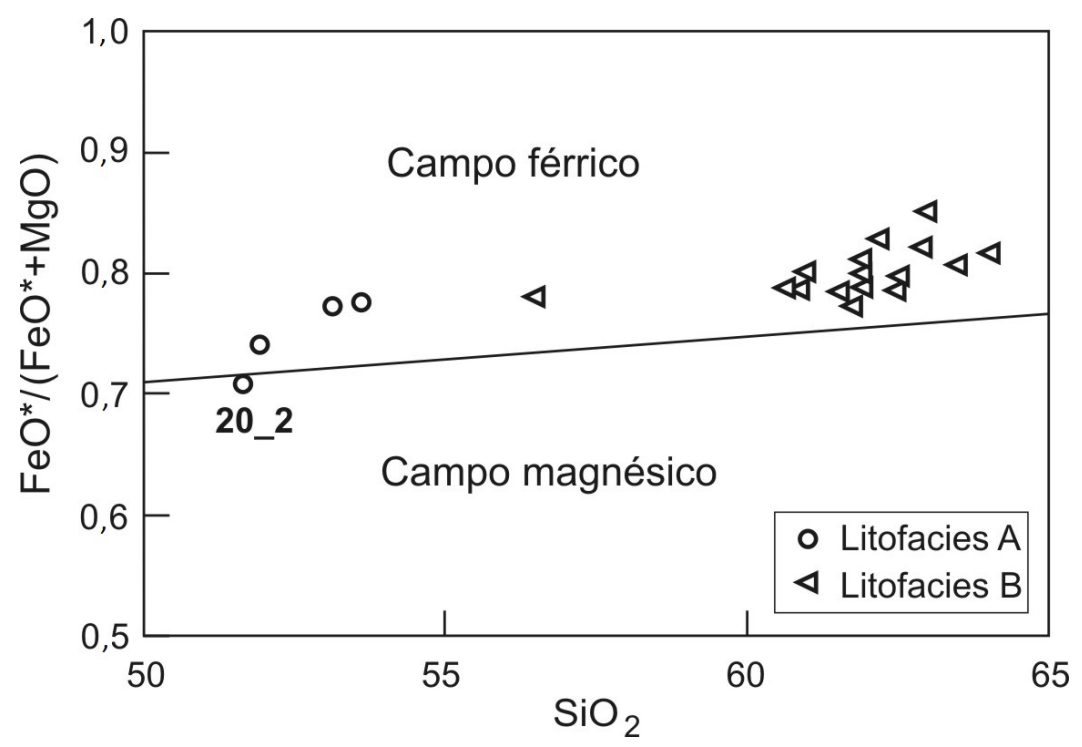

FIG. 6. Clasificación química de las muestras de la parte meridional del plutón Chinquilchoro en el diagrama $\mathrm{FeO} * /(\mathrm{FeO} *+\mathrm{MgO})$ versus $\mathrm{SiO}_{2}$ (\% en peso). El asterisco significa que todo el hierro disponible está expresado en su forma ferrosa.

Las rocas del plutón Chinquilchoro constituyen la parte oriental de una extensa franja de afloramientos que se expone principalmente a lo largo de la cordillera de Domeyko $\left(22^{\circ}\right.$ a $\left.27^{\circ} \mathrm{S}\right)$ y que son la manifestación del magmatismo extrusivo e intrusivo del Paleozoico superior en el norte de Chile (Maksaev et al., 2014; Niemeyer, 2017). Son comparables con stocks de monzodioritas y monzodioritas cuarcíferas que intruyen a las rocas de la Formación La Tabla, del Carbonífero-Pérmico, en el sector de Imilac (Urzúa, 2009).

El carácter férrico del plutón Chinquilchoro sugiere un emplazamiento en ambiente tectónico anorogénico (Frost et al., 2001). Efectivamente, las rocas del Paleozoico superior en la cordillera de Domeyko han sido asignadas a un ambiente tectónico extensional (Breitkreuz et al., 1989; Breitkreuz y Zeil, 1994), donde posiblemente la litofacies de borde del plutón Chinquilchoro habría formado parte de un anillo en la base de una caldera volcánica (Bonin, 2007). Al respecto, en la cordillera Frontal de Argentina, la secuencia equivalente, del denominado Grupo Choiyoi, habría evolucionado durante el Pérmico medio desde un sistema de arco a un régimen tectónico progresivamente extensional relacionado con subducción en retroceso (rollback), Rocher et al. (2015).

\section{Agradecimientos}

Se agradece a L. Hernández por las determinaciones con la microsonda. Asimismo, los autores agradecen la revisión crítica del manuscrito por parte de J. Muñoz y W. Vivallo. Los dibujos fueron realizados por L. Jofré.

\section{Referencias}

Améglio, L.; Vigneresse, J.L. 1999. Geophysical Imaging of the Shape of Granitic Intrusion at Depth: A Review. In Understanding Granites: Integrating New and Classical Techniques (Castro, A.; Fernández, C.; Vigneresse, J.L.; editors). Geological Society, Special Publication 168: 39-54. London.

Barbey, P. 2009. Layering and schlieren in granitoids: A record of interactions between magma emplacement, crystallization and deformation in growing plutons. Geologica Belgica 12 (3-4): 109-133.

Bonin, B. 2007. A-type granites and related rocks: Evolution of a concept, problems and prospects. Lithos 97: 1-29.

Breitkreuz, C.; Bahlburg, H.; Delakowitz, B.; Pichowiak, S. 1989. Paleozoic volcanic events in the Central Andes. Journal of South American Earth Sciences 2 (2): 171-189.

Breitkreuz, C.; Zeil, W. 1994. The Late Carboniferous to Triassic Volcanic Belt in Northern Chile. In Tectonics of the Southern Andes. Structure and Evolution of an 
Active Continental Margin (Reutter, K.J.; Scheuber, E.; Wigger, P.J.; editors). Springer: 277-292. Berlín.

Frost, B.R.; Barnes, C.G.; Collins, W.J.; Arculus, R.J.; Ellis, D.J.; Frost, C.D. 2001. A Geochemical Classification for Granitic Rocks. Journal of Petrology 42 (11): 2033-2048.

Frost, C.D.; Frost, B.R. 2011. On Ferroan (A-type) Granitoids: their Compositional Variability and Modes of Origin. Journal of Petrology 52 (1): 39-53.

Heinrich, E.W.M. 1972. Petrografía Microscópica. Segunda edición. Editorial Omega: 320 p. Barcelona.

Maksaev, V.; Munizaga, F.; Tassinari, C. 2014. Timing of the magmatism of the paleo-Pacific border of Gondwana: U-Pb geochronology of Late Paleozoic to Early Mesozoic igneous rocks of the north Chilean Andes between $20^{\circ}$ and $31^{\circ} \mathrm{S}$. Andean Geology 41 (3): 447-506. doi: 10.5027/andgeoV41n3-a01.

Marsh, B.C. 2015. Magma Chambers. In The Encyclopedia of Volcanoes. Elsevier, Chapter 8: 185-201. doi: 10.1016/B978-0-12-385938-9.00008-0.

Nédélec, A.; Bouchez, J.L. 2015. Zoning in granite plutons. In Granites: Petrology, Structure, Geological Setting, and Metallogeny. Oxford University Press: 335 p. Oxford.

Niemeyer, H. 1989. El Complejo Ígneo-Sedimentario del Cordón de Lila, Región de Antofagasta: significado tectónico. Revista Geológica de Chile 16 (2): 163-181. doi: 10.5027/andgeoV16n2-a03.
Niemeyer, H. 2013. Geología del Área Cerro Lila-Peine, Región de Antofagasta. Servicio Nacional de Geología y Minería, Carta Geológica de Chile, Serie Geología Básica 147: 39 p., 1 mapa escala 1: 100.000. Santiago.

Niemeyer, H. 2017. Geología de la Era paleozoica en el Norte de Chile. Ediciones Universitarias de la Universidad Católica del Norte: 114 p. Antofagasta.

Philpotts, A.R. 1967. Origin of certain iron-titanium oxide and apatite rocks. Economic Geology 62 (3): 303-315.

Rocher, S.; Vallecito, G.; Castro de Machuca, B.; Alasino, P.H. 2015. El Grupo Choiyoi (Pérmico tempranomedio) en la Cordillera Frontal de Calingasta, San Juan, Argentina: Volcanismo de arco asociado a extensión. Revista Mexicana de Ciencias Geológicas 32 (3): 415-432.

Shelley, D. 1993. Igneous and Metamorphic Rocks under the Microscope. Chapman and Hall: 445 p. London.

Streckeisen, A. 1974. Rocas plutónicas. Clasificación y nomenclatura recomendada por la Unión Internacional de Ciencias Geológicas (I.U.G.S.). Subcomisión en sistemática de rocas ígneas. Traducido al Castellano de Neues Jahrbuch für Mineralogie, Abhandlungen, Instituto de Investigaciones Geológicas 107: 144-240. Santiago.

Urzúa, F. 2009. Geology, geochronology and structural evolution of La Escondida copper district, northern Chile. Ph.D. Thesis (Unpublished), University of Tasmania: 486 p. Hobart.

Manuscript received: July 13, 2020; revised/accepted: December 15, 2020; available online: January 29, 2021. 